\title{
Perception of pictorial depth cues by pigeons
}

\author{
SHERI L. REID and MARCLA L. SPETCH \\ University of Alberta, Edmonton, Alberta, Canada
}

\begin{abstract}
Pigeons were trained to discriminate pictures of intact objects from pictures of objects in which both depth from shading and depth from perspective cues were manipulated. Depth from shading was manipulated either by scrambling or by removing three-dimensional shading cues. Depth from perspective was manipulated either by presenting pictures of objects with a two-dimensional outline (i.e., a square) or with a three-dimensional outline (i.e., a cube). Transfer tests with novel images suggest that pigeons perceive and utilize both types of pictorial depth cues. The implications of these results for our understanding of picture perception in pigeons are discussed.
\end{abstract}

Numerous studies in which the perceptual and cognitive abilities of pigeons were investigated have used pictorial displays (categorization: Edwards \& Honig, 1987; Hernstein \& Loveland, 1964; Wasserman, Kiedinger, \& Bhatt; 1988; object recognition: Kirkpatrick-Steger, Wasserman, \& Biederman, 1996; Wasserman et al., 1996; White, Alsop, \& Williams, 1993; and spatial relations: Spetch, Kelly, \& Lechelt, 1998; Spetch \& Wilkie, 1994; Wilkie, Willson, \& MacDonald, 1992). Much of this research is predicated on the assumption that similar cognitive processes underlie responding to both two-dimensional (2-D) and three-dimensional (3-D) stimuli and that the third dimension is adequately represented by pictorial depth cues. However, the question of whether pictorial stimuli do in fact adequately represent 3-D objects and scenes for nonhuman animals has received comparatively little empirical investigation.

Evidence from object-picture equivalence studies suggests that pigeons perceive pictures as representations of real objects. Pigeons trained to discriminate simple geometric objects transfer responding to photographs of training objects (Cabe, 1976; Lumsden, 1977), and pigeons trained to categorize real objects (food and junk items) transfer categorical responding to photographs of both training and novel objects (Delius, 1992; Watanabe, 1993, 1997). Furthermore, birds have been shown to recognize pictures of conspecifics (Trillmich, 1976). Together, these studies suggest that, under certain conditions, nonhuman animals are capable of recognizing the equivalence between 3-D objects and their 2-D pictorial representations.

Although object-picture equivalence studies are able to demonstrate transfer between objects and pictures, it is not certain to what extent these findings reflect a capacity

The research presented in this paper was also presented at the International Conference on Comparative Cognition in Melbourne, FL (March 1997). This research was supported by an operating grant from the Natural Sciences and Engineering Research Council of Canada to M.L.S. We thank D. Kelly for assistance with the research. Correspondence concerning this article should be addressed to $M$. L. Spetch, Department of Psychology, University of Alberta, Edmonton, AB, T6G 2E9 Canada (e-mail: mspetch (apsych.ualberta.ca). to perceive three-dimensionality in 2-D displays, as compared with 2-D feature extraction. Moreover, these studies do not address the question of which features are used to extract three-dimensionality in 2-D displays. A number of depth cues are available for the recovery of the three-dimensionality of objects and scenes in pictures, such as occlusion, linear perspective, and shading. The question of which pictorial depth cues are extracted and utilized by animals has only recently begun to attract experimental investigation.

A growing number of studies suggest that several species of animals other than humans are able to complete 2-D representations of partly occluded objects. Lea, Slater, and Ryan (1996) imprinted chicks to partially occluded triangles and tested whether they would choose to associate with a complete triangle or a fragmented one. The chicks consistently chose to associate with the complete triangles, suggesting that the birds were responding to the underlying unity of the partly occluded training objects. Regolin and Vallortigara (1995) reported similar findings with chicks imprinted on whole objects and tested with occluded versus fragmented objects. Completion of partly occluded 2-D objects has also been reported for mice (Kanizsa, Renzi, Conte, Compostela, \& Guerani, 1993) and monkeys (Osada \& Schiller, 1994). Pigeons, in comparison, have shown little evidence of amodal completion in studies using line drawing stimuli. Cerella (1980) trained pigeons to discriminate pictures of geometric forms as well as cartoon characters and tested whether they would choose partially occluded or fragmented versions of training objects. In both instances, pigeons treated the partially occluded and fragmented forms alike. Similarly, when trained to categorize pictorial figures as complete or incomplete and tested with novel examples, pigeons responded to occluded objects as though they were incomplete (Sekuler, Lee, \& Shettleworth, 1996). These results suggest that, for line drawings at least, pictorial depth cues from occluding boundaries do not adequately represent three-dimensionality for pigeons.

A number of animal species have also been shown to perceive the Ponzo illusion generated from pictorial depth cues (horses, Timney \& Keil, 1996; monkeys, Bayne \& 
Davis, 1983; and pigeons, Fujita, D. S. Blough, \& P. M. Blough, 1991, 1993). The illusion occurs when two parallel bars are located between two converging lines. To humans, the bar near the apex of the lines looks longer than the bar further away. That animals are also susceptible to this illusion suggests that they are able to utilize depth from perspective cues and that it is likely that they share common perceptual processes with humans.

Fewer investigations have been made into animals' perceptual processing of depth from shading cues in pictorial stimuli. Almost three decades ago, Hershberger (1970) trained chicks to discriminate convex and concave dents in an aluminum panel and found that the chicks' responding transferred to photographs of the training dents, suggesting some processing of depth from shading information present in photographic stimuli.

The present experiment was designed to determine whether pigeons could use both shading cues and perspective cues to extract information about three-dimensionality from pictorial representations of objects. Accordingly, we trained pigeons on a simultaneous choice task to discriminate digitized images of 3-D objects from digitized images of objects in which depth cues from shading or perspective were either absent or altered. To discourage the birds from solving the discrimination on the basis of simple 2-D features (e.g., a particular color or shape), we trained the pigeons with a large set of objects that differed in specific local features. To rule out the possibility that the birds might nevertheless have memorized specific features of the large set of positive and negative objects, all of our evidence for the pigeons' ability to extract three-dimensionality from pictorial stimuli comes from nonreinforced transfer tests with images of novel objects.

\section{METHOD}

\section{Subjects}

The subjects were 4 adult silver king pigeons that had previously served as subjects in touch screen chambers in spatial search tasks involving simple graphic stimuli. All the birds were naive with respect to the present discrimination and stimuli used. The birds were housed in large individual cages under a 12:12-h light:dark cycle and maintained at approximately $85 \%$ of their free-feeding body weights. Food was obtained during and after experimental sessions. Water and grit were available ad lib in the home cages.

\section{Apparatus}

The experimental chamber was a modified BRS/LVE operant chamber equipped with a color monitor (Zenith 1492) and an infrared touchframe (Carroll Touch, 1492 Smart Frame). A $28 \times 20 \mathrm{~cm}$ monitor opening was cut into the back wall of the chamber, $9 \mathrm{~cm}$ above the floor. A thin sheet of Plexiglas covered the monitor screen, and spacers were used to recess the touch-frame by approximately $3 \mathrm{~cm}$ from the monitor opening and to separate the frame from the monitor by approximately $1.5 \mathrm{~cm}$. The chamber contained two BRS/LVE grain hoppers, one on each of the side walls, $8 \mathrm{~cm}$ from the back wall and $9 \mathrm{~cm}$ from the floor. Lamps located within each feeder were used to illuminate feeder presentations. Photocells in each hopper measured head entries into the hopper

Experimental contingencies and recording of peck coordinates were controlled by a microcomputer located in an adjacent room. The touchframe was programmed to detect individual pecks (i.e., detection of a
}

beam break, then a return to unbroken beams before another peck would be recorded)

\section{Stimuli}

Images of objects were created from a large set of real objects. The objects were constructed out of plastic, cardboard, or metal and differed from one another in (1) basic geometric shape (e.g., cubes, spheres, cylinders, cones, or rectangular and triangular prisms), (2) size and dimensions, (3) color, and (4) the orientation from which they were filmed. The objects were videotaped with a camcorder, and then played into a computer, using a frame grabber system (Creative Labs Video Blaster). The still frames were then saved in GIF format (Compuserve, Inc.) and edited, using Photofinish software (Zsoft).

Four types of objects were created. (1) 3-D objects were unedited images of real objects, filmed to reveal their 3-D shapes. Two cues to three-dimensionality were present: the 3-D shape of the object (depth from linear perspective) and depth from shading cues. (2) Perspectivecue-only objects were created to provide depth from linear perspective cues but no depth from shading cues. These were filmed objects having a 3-D shape and edited to have either a uniform fill or scrambled shading cues. The linear perspective cue to three-dimensionality consisted of the nonparallel lines defining the outline of each object. The inclusion of objects with scrambled shading cues served to prevent discrimination based solely on the presence or absence of shading. Scrambling of the cues was accomplished by moving, breaking into parts, and/or rotating the shading cues present in the original object. Shaded areas were placed variously either next to an edge or nearer the center of the objects. (3) Shading-cue-only objects were objects created with graphic software (Photofinish) to have a 2-D shape and 3-D shading cues. The 3-D shading cue for square, rectangle, and some triangle shapes was created by adding a Y-junction within the boundaries of the object and filling it with a color different from the rest of the object. For all other shapes, 3-D shading was created by adding a different band of color along an edge of the object. (4) 2-D objects were objects created with graphic software (Photofinish) that had a 2-D shape and either a uniform fill or scrambled shading cues. Both 3-D shape and 3-D shading cues were absent.

A large number of stimulus displays were created for training and testing. Each stimulus display consisted of a digitized image of two objects centered side by side within rectangular outlined areas. In some images, the 3-D object was presented together with an altered version of the same object. In other images, the 3-D object was presented together with a different altered or 2-D object. The 3-D object was equally often on the left or right. See Figures 1 and 2 for examples of the training and testing objects.

\section{General Procedures}

Training involved a simultaneous discrimination procedure, with 3D objects as the positive stimulus ( $\mathrm{S}+$ ) and 2-D or altered objects as the negative stimulus $(S-$. Each trial began with the presentation of a stimulus display that remained on until the pigeon made a single peck within one of the rectangular areas containing the two objects. On training and baseline trials, if the area containing a 3-D object $\left(\mathrm{S}^{+}\right)$was pecked, a food reward (2-sec access to a randomly selected food hopper) was presented with a probability of 0.5 (i.e., a $50 \%$ reinforcement schedule for correct choices was in effect). If the area containing the $S-$ object was pecked, the trial ended without reinforcement, and a correction procedure was initiated. During correction procedures, the same picture pair was re-presented until the pigeon made a correct choice. Correction trials were not used in the determination of accuracy. All the test trials were conducted in extinction: The first peck within either of the areas containing an object terminated the trial without reinforcement, whether or not a correct choice was made. Incorrect choices on test trials did not result in a correction trial. The intertrial interval (ITI) was $2 \mathrm{sec}$, unless otherwise stated. During ITls, the monitor was blank. Sessions were conducted at approximately the same time each day, 5 or 6 days per week. Sessions lasted until all scheduled trials were completed or for a maximum of $1 \mathrm{~h}$

Pretraining. All the birds had previous experience in touch-screen tasks and were placed on autoshaping to reestablish reliable pecking. 

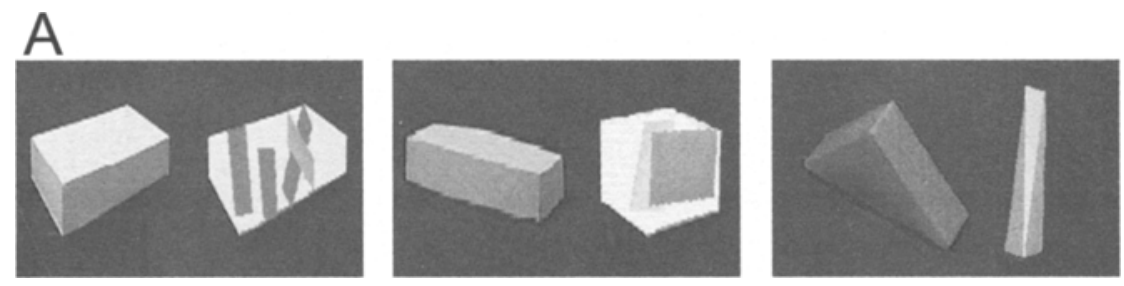

B
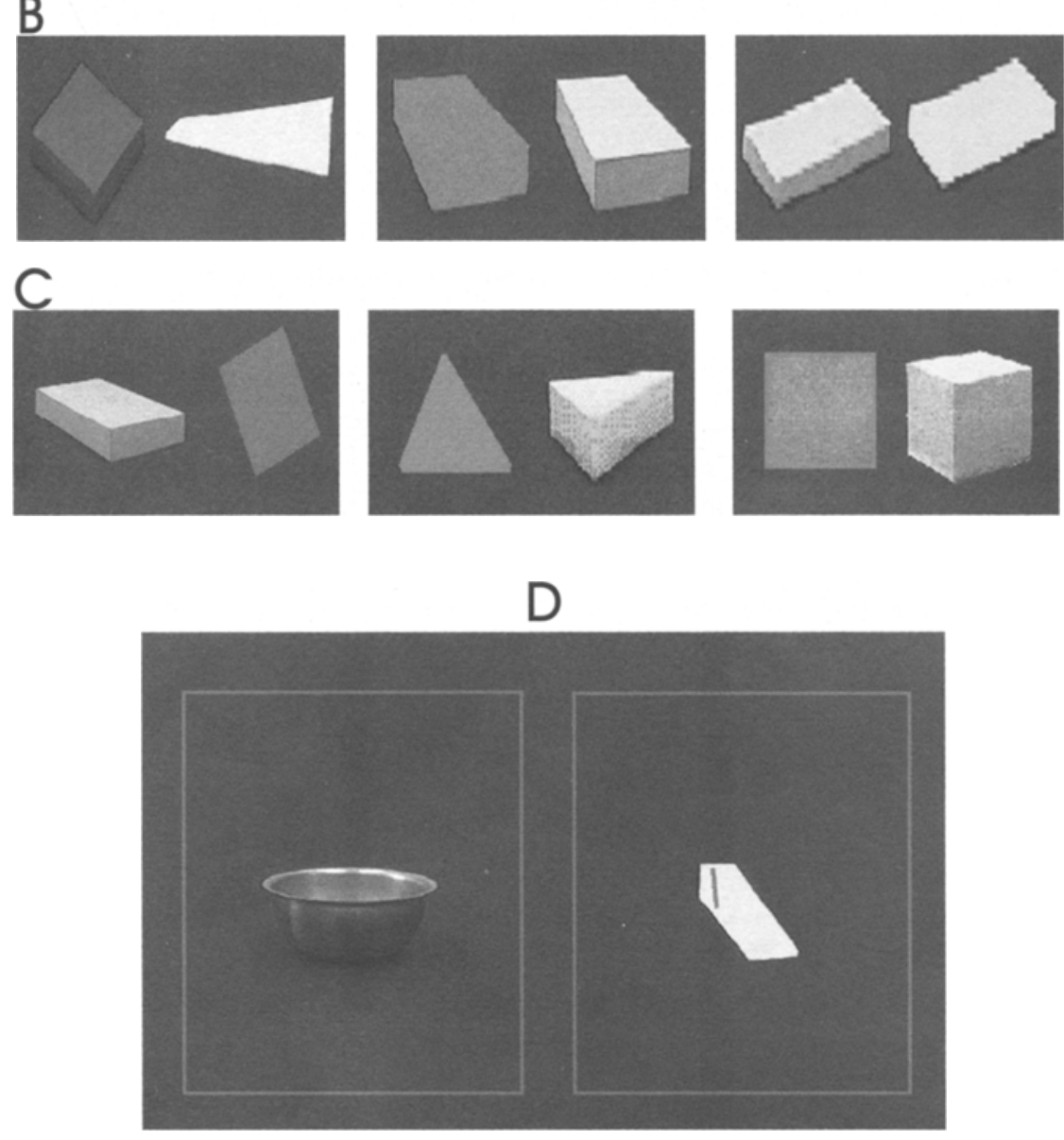

Figure 1. Examples of training stimulus objects (Rows $A, B$, and $C$ ) and a training stimulus display (Row D). One member of each stimulus pair is an image of a three-dimensional (3-D) object, and the other member is an image of either an altered 3-D object or a twodimensional (2-D) object. In Row $A$, the altered object in each pair is a perspective-cue-only training object (depth from linear perspective cues present, depth from shading cues absent) with shading cues scrambled. In Row B, the altered object in each pair is a perspective-cueonly training object with shading cues absent. Pictures in Row $C$ represent 3-D versus 2-D training objects. The picture in Row $D$ is an example showing how object pairs were displayed on the computer screen during training and testing. Images were displayed in color and were sharper than they appear here.

Each autoshaping trial began with the presentation of one of the training stimulus displays. The stimulus display remained on for $8 \mathrm{sec}$ or until a peck in the area containing the 3-D object was recorded. The completion of either event resulted in access to a food hopper. Trials were separated by a 40 -sec ITI.

Training and testing. In Phase 1 , the pigeons were trained to discriminate $243-\mathrm{D}$ objects from 24 perspective-cue-only objects (one third of these perspective-cue-only objects had shading cues removed, and two thirds had scrambled shading cues). The 3-D objects were designated as positive, and the perspective-cue-only objects were desig- nated as negative. Each session consisted of 120 trials, with each stimulus display being presented five times. The pigeons were trained for a minimum of 20 sessions or until they reached an average of $85 \%$ correct responses over 6 consecutive sessions. All birds reached criterion within 20 sessions.

Once the birds met criterion, nonreinforced transfer tests were conducted with novel intact objects and novel manipulated objects. During the first series of transfer testing, 30 test images each presented one novel 3-D object together with one novel perspective-cue-only object (one-third of these perspective-cue-only objects had shading cues re- 

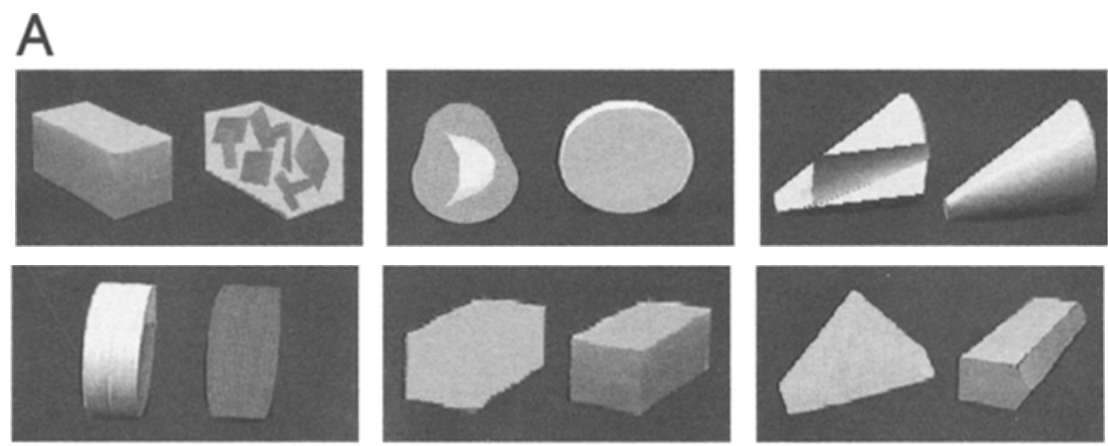

B
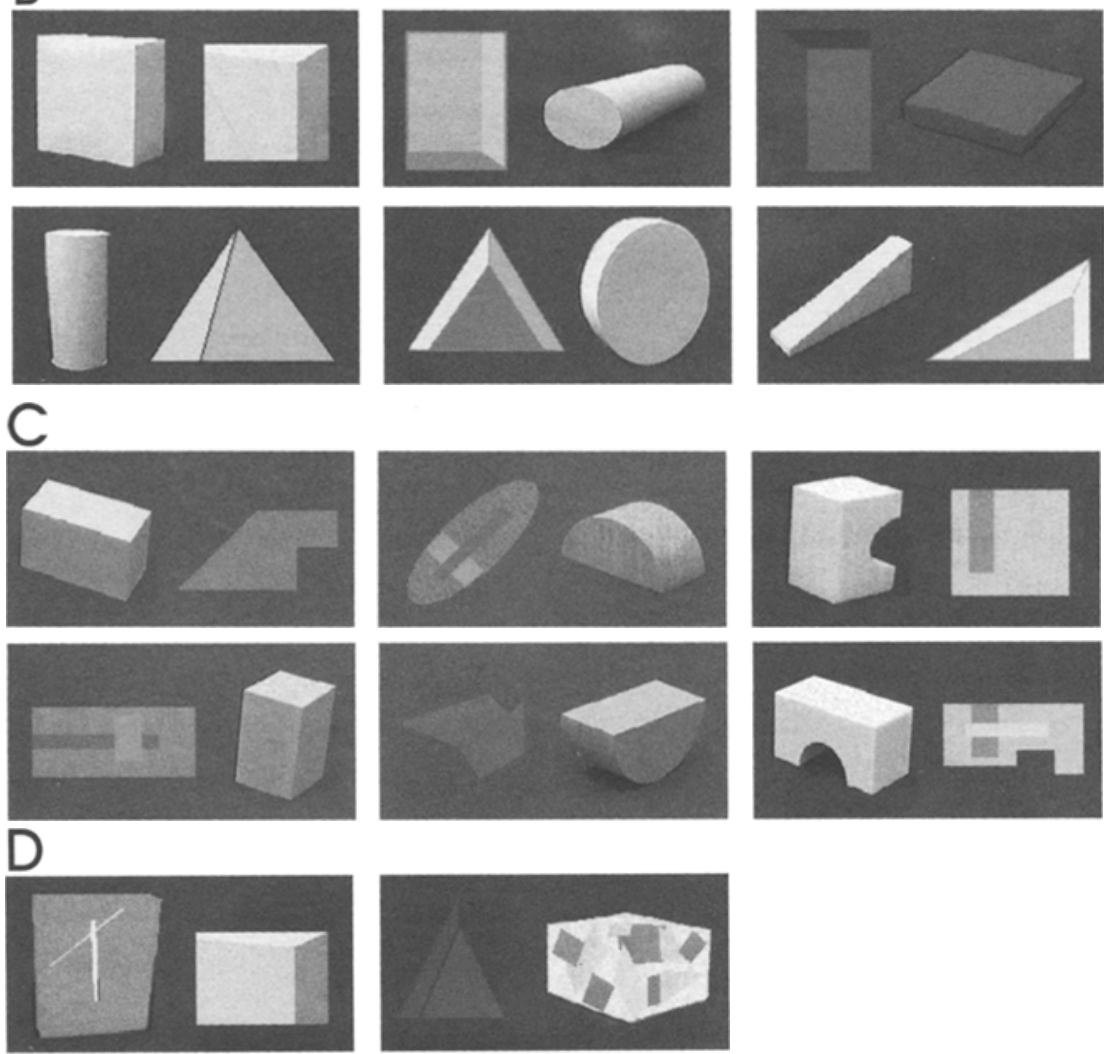

Figure 2. Examples of test stimulus objects. One member of each stimulus pair is an image of a three-dimensional (3-D) object, and the other member is an image of either an altered 3-D object or a two-dimensional (2-D) object. Pictures in the rows labeled $A$ show a 3-D object together with a perspective-cue-only object. The perspective-cue-only objects in the first row have scrambled shading cues, whereas those in the second row have shading cues absent. Pictures in the rows labeled B are 3-D objects versus shading-cue-only objects (depth from linear perspective cues absent, depth from shading cues present). Pictures in the rows labeled $C$ represent 3-D versus 2-D objects. Row D shows the two shading-cue-only versus perspectivecue-only stimulus pairs. Object pairs were displayed on the computer sereen as shown in Row D of Figure 1. Images were displayed in color and were sharper than they appear here.

moved, and two thirds had scrambled shading cues). In addition, 10 test images displayed novel 3-D objects together with novel shading-cueonly objects. Test sessions were divided into five blocks that each presented 32 stimulus displays: 16 reinforced training stimulus displays, 8 nonreinforced training stimulus displays that served as controls, 6 test displays in which novel 3-D objects were paired with novel perspectivecue-only objects, and 2 test displays in which novel 3-D objects were paired with novel shading-cue-only objects. Each session consisted of 128 trials, so that each display was presented four times. Three test ses- sions were conducted for each block of testing. During a second transfer test, a perspective-cue-only object was presented together with a shading-cue-only object on test trials. This test pitted depth cues from perspective against depth cues from shading. Two test displays were presented four times each, interspersed among control and baseline trials, for three sessions.

In Phase 2, the pigeons were trained to discriminate pictures of 3-D objects from pictures of 2-D objects in which both perspective and shading cues were absent. The training set and procedures were the 
same as in Phase I, except that 8 new stimulus displays were added to the training set. These new stimulus displays presented novel 3-D objects paired with novel 2-D objects. All of the 2-D objects had uniform fill (no shading). Each session was 128 trials, with each display being presented four times. The pigeons took between 7 and 20 sessions to reach a criterion of $85 \%$ correct responding averaged over 6 consecutive sessions. The birds were then tested with 8 new stimulus displays, each presenting a novel 3-D object and a novel 2-D object. In each session of testing, 32 stimulus displays were presented: 16 reinforced training stimulus displays, 8 nonreinforced training stimulus displays that served as controls, and 8 novel nonreinforced test displays. Each session consisted of 128 trials, with each display being presented four times. Testing continued for three sessions.

Phase 3 was a replication of Phase 2, except that some of the 2-D objects used in training and testing contained scrambled shading cues. Only 3 pigeons served in Phase 3, because 1 bird became ill and had to be dropped from the experiment. During training, 2 new stimulus displays containing 2-D objects with scrambled shading were added to the training set. All the birds met the accuracy criterion of $85 \%$ averaged over six sessions within the minimum six sessions. The birds were then tested with 8 new stimulus displays, each presenting a novel 3-D object and a novel 2-D object. Half of the novel 2-D objects had uniform fill, and half had scrambled shading. In each session of testing, 32 stimulus displays were presented: 16 reinforced training stimulus displays, 8 nonreinforced training stimulus displays that served as controls, and 8 novel nonreinforced test displays. Each session consisted of 128 trials, with each display being presented four times. Testing continued for three sessions.

\section{RESULTS}

An alpha level of .05 was used for all statistical tests. Accuracy scores for control (nonreinforced previously trained images) and test trials are shown in Figure 3. Despite the fact that all the test objects were novel and all the test trials were nonreinforced, accuracy was above chance level $(50 \%)$ on each type of test. For each test type, a one-tailed $t$ test was used to confirm that accuracy was significantly higher than chance.

During Phase 1 (top panel of Figure 3), accuracy in selecting the 3-D object was significantly above chance on tests in which the incorrect object contained perspective cues only $[t(3)=4.17]$, as well as on tests in which the incorrect object contained shading cues only $[t(3)=$ 3.63]. Moreover, the pigeons' accuracy was greater than chance in choosing 3 -D objects over perspective-cue-only objects that had scrambled shading cues $[t(3)=3.81]$. On tests that pitted a perspective-cue-only object against a shading-cue-only object, choice of the shading-cue-only object was significantly above $50 \%[t(3)=5.61]$.

During both Phase 2 and Phase 3 (bottom panel of Figure 3), the pigeons chose the 3-D object over the 2-D object significantly more often than would be expected by chance [Phase 2, $t(3)=9.70$; Phase $3, t(2)=4.63$ ]. A detailed analysis of Phase 3 test results further revealed that the pigeons were above chance in choosing the 3-D object over 2-D objects that had scrambled shading cues $[t(2)=4.28]$

An analysis of variance on the various types of tests in Phase 1 revealed a significant overall effect of trial type $[F(3,9)=32.53]$. A test of multiple comparisons (Tukey's HSD) revealed that performance on control trials was significantly higher than performance on each of the three types of tests. In addition, accuracy on the shading versus perspective cue tests was higher than accuracy on the shading-cue-only tests. This indicates that the birds were more accurate in choosing a shading-cue-only object over a perspective-cue-only object than in choosing a 3-D object over a shading-cue-only object.

\section{DISCUSSION}

The results from this experiment show that pigeons are able to discriminate pictures of 3-D objects from pictures of 2-D objects and from pictures of objects in which depth from shading and depth from perspective cues are either absent or altered. Three aspects of the results make it unlikely that the pigeons solved the task without attending to the pictorial depth cues. First, all the objects presented on test trials were novel objects that differed from the training objects in any of several ways (color, shape, orientation, texture, size, etc.). Thus, memorization of specific features of the training objects should not allow the birds to respond accurately on test trials. Second, all test trials were nonreinforced, so the above-chance accuracy could not reflect new learning. In fact, there was some tendency for accuracy to decrease over the three sessions of testing. Third, the birds could not solve the task by using the presence or absence of shading as a simple cue to three-dimensionality, because two thirds of the perspective-cue-only objects and one half of the 2-D objects of Phase 3 had scrambled shading cues. Overall, the discrimination of pictorial depth cues suggests that the third dimension is adequately represented in digitized images for pigeons.

The results of the perspective-cue-only and the shading-cue-only tests showed that pigeons could discriminate objects having two depth cues (i.e., the 3-D objects that had both 3-D shape and 3-D shading) from objects having one only depth cue (either the 3-D shape or the 3-D shading). This finding indicates that the pigeons could use both types of pictorial cues to determine three-dimensionality. Specifically, on perspective-cue-only tests, the critical difference between the 3-D object and the incorrect object was that only the 3-D object had shading that was indicative of three-dimensionality. The above-chance accuracy indicates that they were sensitive to these shading cues. Similarly, on the shading-cue-only tests, the critical difference between the 3-D object and the incorrect object was that only the 3-D object had an outline shape that was indicative of three-dimensionality. The above-chance accuracy on this test indicates that they were sensitive to these perspective cues. However, accuracy was low on these tests, compared with that observed on tests in which the birds discriminated 3-D objects from 2-D objects (i.e., from objects with neither depth cue), perhaps because the pigeons perceived some three-dimensionality in objects having only one depth cue.

Although the similarity of performance on perspective-cue-only and shading-cue-only tests might suggest that perspective and shading cues are equally useful cues to three-dimensionality, it should be noted that the birds had been trained to discriminate 3-D objects from perspectivecue-only objects but had never been trained to discriminate 3-D objects from shading-cue-only objects. Similarly, although the pigeons showed significant choice of the shading-cue-only objects over the perspectivecue-only objects, we cannot conclude that this reflects dominance of shading cues over perspective cues, because it could instead reflect their training history: Only perspective-cue-only objects served as S- objects during training. Thus, we have demonstrated that pigeons are sensitive to both kinds of pictorial depth cues, but we cannot make conclusions about their relative dominance independent of training experiences.

Although the finding that pigeons are sensitive to depth from shading cues has not been previously demonstrated in any studies that we are aware of, the finding that pigeons are sensitive to depth from perspective cues is supported by previous studies in which pigeons have been shown to be sensitive to the Ponzo illusion generated from pictorial depth from perspective cues (Fujita et al., 1991, 1993).

In contrast, studies investigating the perceptual completion of partially occluded objects report that interposition cues do not adequately represent depth relations in pictures for pigeons. Both Cerella (1980) 

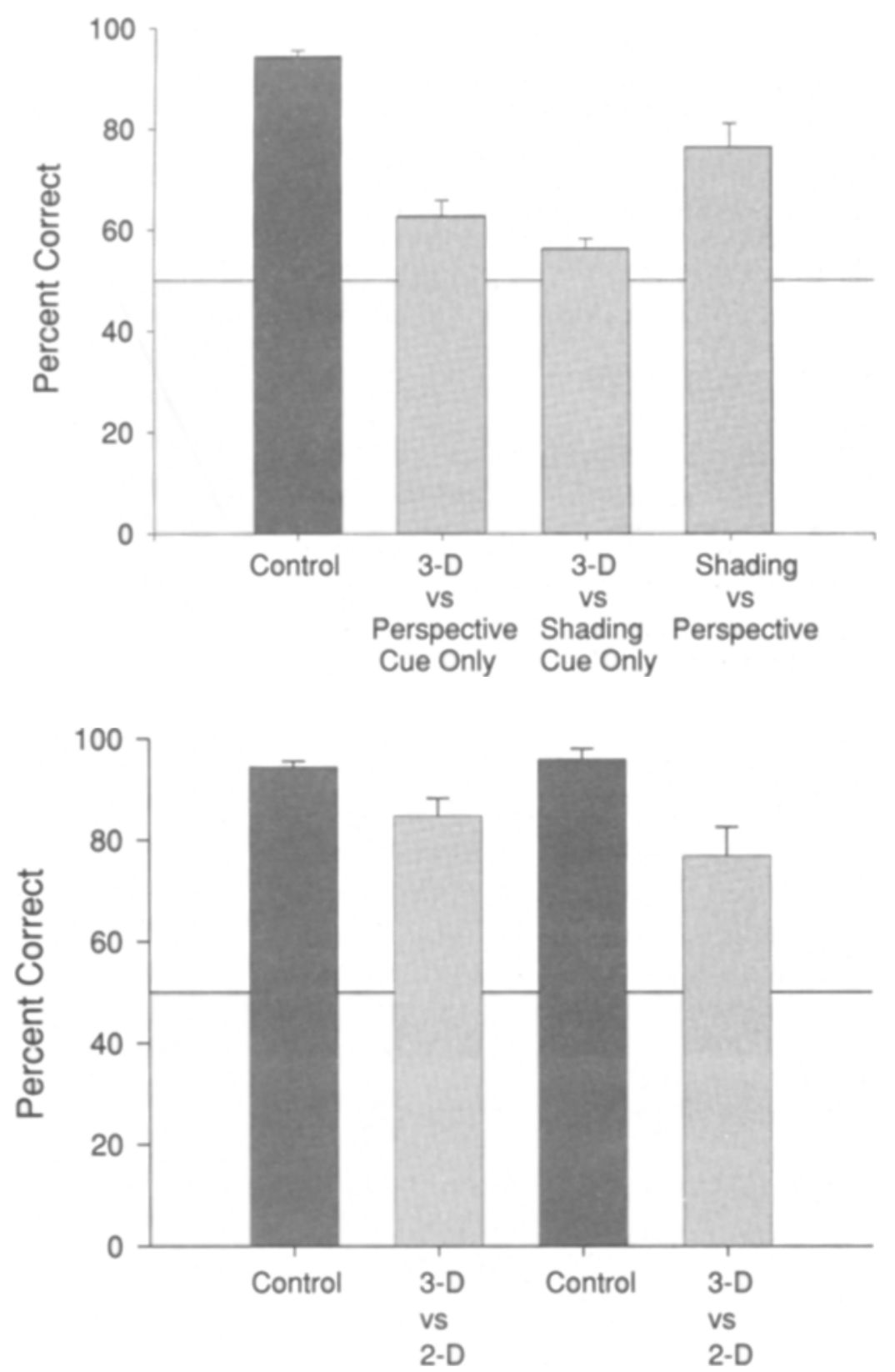

Figure 3: Accuracy on control trials (nonreinforced trials with previously trained images) and on nonreinforced test trials with completely novel objects. The top panel shows the results from Phase 1 of the experiment. Bars $1-3$ represent the percentage of correct responses to three-dimensional (3-D) objects. Bar 4 represents the percent of responses to shading-cue-only objects. The bottom panel shows the results from Phases 2 and 3. Bars 1 and 2 represent the percentage of correct responses to 3-D objects in Phase 2. Bars 3 and 4 represent the percentage of correct responses to 3-D objects during Phase 3.

and Sekuler et al. (1996) have reported that pig eons respond to partially occluded objects as fragments of complete objects. Furthermore, pigeons trained to discriminate line drawings of cartoon characters respond similarly to scrambled versions of the training stimuli (Cerella, 1980). These results suggest that picture perception in the pigeon is based on the identification of local features rather than on the identification of global features for three-dimensionality. In each of these studies, however, unshaded line drawings of artificial stimuli were used to represent objects and the figure-ground relationships between them.
Whether pigeons respond to fragmented and scrambled versions of objects as being equivalent to complete objects when photographic stimuli are used is currently being investigated in our laboratory.

The present study is the first that we are aware of to provide direct ev. idence that pigeons are able to utilize information from both depth from shading and depth from perspective pictorial cues to discriminate 3-D objects from objects in which 3-D cues are either absent or altered. Although we are not able to conclude that pigeons perceive pictures as 3-D in the same manner that humans do, our results suggest that the pi- 
geons did not discriminate the pictures on the basis of the presence of a simple local cue. First, all of the objects presented on test trials differed from the training ones on several features (i.e., color, shape, orientation, texture, and size). And second, when performance on individual stimulus objects was examined, we did not find any systematic differences in accuracy on the basis of the presence of a single cue, such as color or shape. Therefore, if the pigeons' performance was based on the learning of local cues, this learning would have to have been based on some complex constellation of local cues. Although no one study is likely to be definitive, our results add to the growing evidence that pigeons are sensitive to the three-dimensionality of objects or scenes presented in pictures.

\section{REFERENCES}

Bayne, K. A. L., \& Davis, R. T. (1983). Susceptibility of rhesus monkeys (Macaca mulatta) to the Ponzo illusion. Bulletin of the Psychonomic Society, 21, 476-478.

CABE, P. A. (1976). Transfer of discrimination from solid objects to pictures by pigeons: A test of theoretical models of pictorial perception. Perception \& Psychophysics, 19, 545-550.

Cerella, J. (1980). The pigeon's analysis of pictures. Pattern Recognition, 12, 1-6.

Delius, J. D. (1992). Categorical discrimination of objects and pictures by pigeons. Animal Learning \& Behavior, 20, 301-311.

EDWARDS, C. A., \& HoNIG, W. K. (1987). Memorization and "feature selection" in the acquisition of natural concepts in pigeons. Learning \& Motivation, 18, 235-260.

Fujita, K., Blough, D. S., \& Blough, P. M. (1991). Pigeons see the Ponzo illusion. Animal Learning \& Behavior, 19, 283-293.

Fusita, K., Blough, D. S., \& Blough, P. M. (1993). Effects of the inclination of context lines on perception of the Ponzo illusion by pigeons. Animal Learning \& Behavior, 21, 29-34.

Hernstein, R. J., \& Loveland, D. H. (1964). Complex visual concept in the pigeon. Science, 146, 549-551.

HershbeRGER, W. (1970). Attached-shadow orientation perceived as depth by chickens reared in an environment illuminated from below. Journal of Comparative \& Physiological Psychology, 73, 407-411.

Kanizsa, G., Renzi, P., Conte, S., Compostela, C., \& Guerani, L. (1993). Amodal completion in mouse vision. Perception, 22, 713-721.

KirkPatrick-Steger, K., Wasserman, E. A., \& Biederman, I. (1996). Effects of spatial rearrangement of object components on picture recognition in pigeons. Journal of the Experimental Analysis of Behavior, 65, 465-475.

Lea, S. E. G., Slater, A. M., \& Ryan, C. M. E. (1996). Perception of object unity in chicks: A comparison with the human infant. Infant Behavior \& Development, 19, 501-504.

LUMSDEN, E. A. (1977). Generalization of an operant response to photographs and drawings/silhouettes of a three-dimensional object at various orientations. Bulletin of the Psychonomic Society, 10, 405-407.

OsADA, Y., \& SCHILler, P. H. (1994). Can monkeys see objects under conditions of transparency and occlusion? Investigative Ophthalmology \& Visual Science, 35, 1664.

Regolin, L., \& Vallortigara, G. (1995). Perception of partly occluded objects by young chicks. Perception \& Psychophysics, 57, 971-976.

Sekuler, A. B., Lee, J. A. J., \& Shettleworth, S. J. (1996). Pigeons do not complete partly occluded figures. Perception, 25, 1109-1120.

Spetch, M. L., Kelly, D. M., \& Lechelt, D. P. (1998). Encoding of spatial information in images of an outdoor scene by pigeons and humans. Animal Learning \& Behavior, 26, 85-102.

SPETCH, M. L., \& WiLKIE, D. M. (1994). Pigeons' use of landmarks presented in digitized images. Learning \& Motivation, 25, 245-275.

Timney, B., \& KeIL, K. (1996). Horses are sensitive to pictorial depth cues. Perception, 25, 1121-1128.

TRILlmich, T. (1976). Learning experiments on individual recognition in budgerigars (Melopsittacus undulatus). Zeitschrift für Tierpsychologie, 41, 372-395.

Wasserman, E. A., Gagliardi, J. L., CoOk, B. R., KirkpatrickSteger, K., Astley, S. L., \& Biederman, I. (1996). The pigeon's recognition of drawings of depth-rotated stimuli. Journal of Experimental Psychology: Animal Behavior Processes, 22, 205-221.

Wasserman, E. A., Kiedinger, R. E., \& Bhatt, R. S. (1988). Conceptual behavior in pigeons: Categories, subcategories, and pseudocategories. Journal of Experimental Psychology: Animal Behavior Processes, 14, 235-246.

WataNaBE, S. (1993). Object-picture equivalence in the pigeon: An analysis with natural concept and pseudoconcept discriminations. Behavioural Processes, 30, 225-232.

WATANABE, S. (1997). Visual discrimination of real objects and pictures in pigeons. Animal Learning \& Behavior, 25, 185-192.

White, K. G., Alsop, B., \& Williams, L. (1993). Prototype identification and categorization of incomplete figures by pigeons. Behavioural Processes, 30, 253-258.

WiLkiE, D. M., Willson, R. J., \& MacDonald, S. E. (1992). Animals' perception and memory for places. In W. K. Honig \& J. G. Fetterman (Eds.), Cognitive aspects of stimulus control (pp. 89-112). Hillsdale, NJ: Erlbaum.

(Manuscript received May 20, 1997; revision accepted for publication February 6,1998 .) 